Accepted (peer-reviewed) version of article

It is authorized for self-archiving after an embargo period of 6 months.

Formatted by the author to enhance readability. 


\title{
The Prehistory of Number Concept
}

\section{Karenleigh A. Overmann, ${ }^{1}$ Thomas Wynn, ${ }^{2}$ and Frederick L. Coolidge ${ }^{3}$}

\begin{abstract}
Carey leaves unaddressed an important evolutionary puzzle: In the absence of a numeral list, how could a concept of natural number ever have arisen in the first place? Here we suggest that the initial development of natural number must have bootstrapped on a material culture scaffold of some sort, and illustrate how this might have occurred using strings of beads.
\end{abstract}

In her discussion of a child's acquisition of the concept of natural number, Carey (2009) makes a strong case that a symbolic place-holder structure is necessary for Quinian bootstrapping. However, Carey's argument leaves an important evolutionary puzzle unaddressed: In the absence of a numeral list, a count list of some kind, or perhaps even language itself, how could a concept of natural number ever have arisen?

Ethnographic and comparative linguistic data suggest that material phenomena can act as scaffolds for the development of a natural number concept. Drawing on a wide range of languages and number systems, both modern and ancient, Menninger (1992) noted that number words first appear in writing and only later in speech, in the form of ordered quantification adjectives up to "three" or "four," a limit consistent with parallel individuation rather than some external system such as finger counting. Quantification adjectives later detach from their objects, and a transition to larger numbers consistent with magnitude representation occurs. Therefore, the purely linguistic evidence would appear to support the necessity of a full-blown linguistic model in support of a concept of natural number. However, Menninger also noted that many people who lack number words can and do arrange objects by one-to-one correspondence, and that tally sticks are "universal” (Menninger, 1992, p. 224).

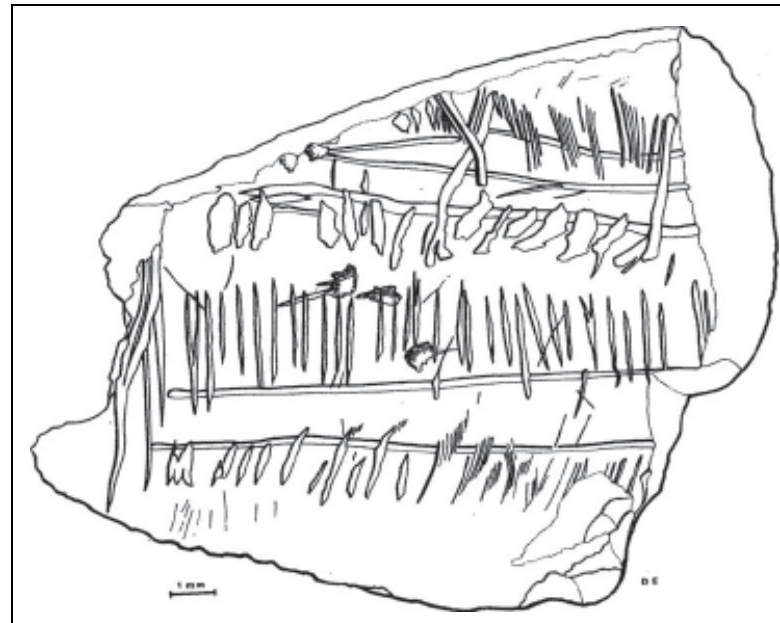

Figure 1. Drawing of the Tossal de la Roca plaque.
There are archaeological reasons for attributing number concept to much older cultures than those with writing. For several thousand years prior to the appearance of writing, Neolithic farmers in the Middle East used an accounting procedure in which individual clay tokens were matched in one-to-one correspondence to individual animals, measures of grain, and so on. The total of the tokens matched the quantity of the commodity (Schmandt-Besserat 1992). Earlier still are items such as the Tossal de la Roca plaque (Fig. 1), which is around 14,000 years old (d'Errico \& Cacho 1994).

Someone engraved this "tally board" with sets of marks, using different tools, at different times. The groups of marks exceed the maximum of parallel individuation. Someone was keeping track of something, and whereas it is possible that it was simply a system of one-to-one correspondence, the use of different tools to add marks suggests some inchoate notion of individuated quantity beyond the limit of parallel individuation. The use of a material object in this case is provocative because it points to an alternative scenario for the emergence of natural number, one based

\footnotetext{
${ }^{1}$ Department of Psychology, University of Colorado at Colorado Springs, Colorado Springs, CO 80918; koverman@uccs.edu.

${ }^{2}$ Department of Anthropology, University of Colorado at Colorado Springs, Colorado Springs, CO 80918; twynn@uccs.edu.

${ }^{3}$ Department of Psychology, University of Colorado at Colorado Springs, Colorado Springs, CO 80918; fcoolidg@uccs.edu.
} 
in extended cognition (Wilson \& Clark 2009), not mental representations of linguistic symbols. We suggest that the initial development of natural number must have bootstrapped on a material culture scaffold of some sort. In place of a symbolic placeholding structure, there were material place-holding structures (Malafouris 2010). There are several possibilities, including tally boards or tokens of some kind, but the earliest example, and the one with the most potential, is the string of beads.

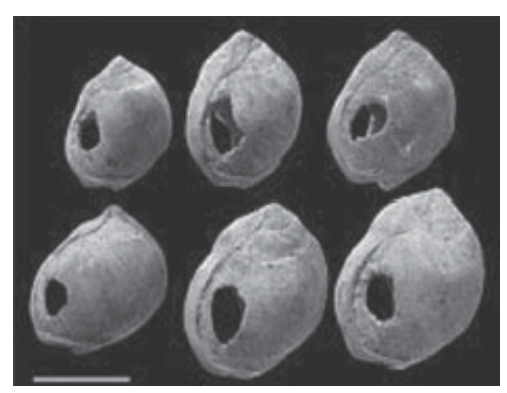

Figure 2. Six of the 40 beads from Blombos Cave.

Archaeology provides a record of beads going back around 100,000 years. The best early examples come from Blombos Cave in South Africa (d'Errico et al. 2005). The beads are small, blue shells with punched holes (Fig. 2).

Wear traces around the hole edges indicate that the beads were strung. The Blombos beads are 77,000 years old; the nearby site of Pinnacle Point boasts similar beads that may be 20,000 years older (Bar-Matthews et al. 2010). The makers were modern human hunters and gatherers. Beads have also been recovered in the 40,000-50,000year time range in Kenya and Turkey, and they are common beginning 30,000 years ago (Ambrose 1998; Kuhn et al. 2009).

A string of beads possesses inherent characteristics that are also components of natural number. There is individuation in the guise of individual beads, ordinality in their invariant sequence on the string, and a material instantiation of $N+1$ : every added bead increases the quantity of beads by the same amount. There is no necessity that people stringing beads understand them in this way, but the potential is there in a very real, tangible sense. And if our bead stringer makes an example with more than three or four beads, he or she will carry individuation beyond the range of parallel individuation. In a sense, the string of beads is a kind of feral number line, without an attendant number concept. When people use a string of beads as a record-keeping device, the beads come to play another role altogether. As Carey puts it: "The critical analogy that provides the key to understanding how the count list represents number is between order on the list and order in a series of sets related by an additional individual" (p. 477, emphasis ours). In the case of the string of beads, the analogy would be between sets in the real world and placeholder beads, which already possess an inherent $N+1$. A true numeral list emerges when people attach individual labels to the various placeholder beads. This material extended cognition scenario has greater natural potential than body-counting systems to scaffold development of natural number. Body-counting systems lack ordinality and lack the natural $N+1$.

We do not know if the initial development of natural number concept took place in this fashion, or indeed even with beads. Our point is that the initial cultural construction of number could most easily have occurred using material objects rather than mental representations. Arguably, such a use of material markers is tantamount to symbol use; beads can stand for other things via one-to-one correspondence, a use preserved in the abacus and rosary. But this strikes us as far less general than the symbolic models Carey uses as examples of Quinian bootstrapping, and therefore more likely as an initial cultural construction.

Material objects with the potential to act as scaffolds for a natural number concept may have been available more or less continuously for perhaps 100,000 years. In cultural circumstances that required the development of more sophisticated numbering, such as formal record-keeping in an economic exchange system, these material scaffolds likely played a crucial role. 


\section{References}

Ambrose, S. H. (1998). Chronology of the Later Stone Age and food production in East Africa. Journal of Archaeological Science, 25(4), 377-392.

Bar-Matthews, M., Marean, C. W., Jacobs, Z., Karkanas, P., Fisher, E. C., Herries, A. I. R., ... Nilssen, P. J. (2010). A high resolution and continuous isotopic speleothem record of paleoclimate and paleoenvironment from 90 to $53 \mathrm{ka}$ from Pinnacle Point on the south coast of South Africa. Quaternary Science Reviews, 29(17-18), 2131-2145.

Carey, S. (2009). The origin of concepts. Oxford: Oxford University Press.

D’Errico, F., \& Cacho, C. (1994). Notation versus decoration in the Upper Palaeolithic: A case-study from Tossal de la Roca, Alicante, Spain. Journal of Archaeological Science, 21(2), 185-200.

D’Errico, F., Henshilwood, C. S., Vanhaeren, M., \& Van Niekerk, K. L. (2005). Nassarius kraussianus shell beads from Blombos Cave: Evidence for symbolic behaviour in the Middle Stone Age. Journal of Human Evolution, 48(1), 3-24.

Kuhn, S. L., Stiner, M. C., Güleç, E., Özer, I., Y1lmaz, H., Baykara, I., ... Suata-Alpaslan, F. (2009). The early Upper Paleolithic occupations at Üçağızlı Cave (Hatay, Turkey). Journal of Human Evolution, 56(2), 87-113.

Malafouris, L. (2010). Grasping the concept of number: How did the sapient mind move beyond approximation? In C. Renfrew \& I. Morley (Eds.), The archaeology of measurement: Comprehending heaven, earth and time in ancient societies (pp. 35-42). Cambridge: Cambridge University Press.

Menninger, K. (1992). Number words and number symbols: A cultural history of numbers. (P. Broneer, Trans.). New York: Dover Publications, Inc.

Schmandt-Besserat, D. (1992). How writing came about. Austin, TX: University of Texas Press.

Wilson, R. A., \& Clark, A. (2009). How to situate cognition: Letting nature take its course. In Philip Robbins \& Murat Aydede (Eds.), The Cambridge handbook of situated cognition (pp. 55-77). New York: Cambridge University Press. 